\title{
Development and characterization of the amidoxime/Europium (III)-chelated complex fibers
}

\author{
C. G. Hu ${ }^{1}$, S. L. Wang ${ }^{2}$, Z. Z. Wu ${ }^{1}$, \\ Z. $\mathrm{ZaO}^{3}$, G. $\mathrm{Li}^{4,5}$
}

\footnotetext{
${ }^{1}$ College of Textile and Clothing Anhui Polytechnic University, Wuhu 241000, P.R.China e-mail: huchenggong2014@163.com

2 Anhui Business College of Vocational Technology, Wuhu 241000, P.R.China

e-mail: wangsunlin2014@163.com

${ }^{3}$ State Key Lab of Advanced Technology for Materials Synthesis and Processing, Wuhan University of Technology,

Wuhan 430070, P.R. China

e-mail: tcligang@suda.edu.cn

${ }^{4}$ National Engineering Laboratory for Modern Silk, College of Textile and Clothing Engineering, Soochow University, Suzhou 215123, P.R.China

${ }^{5}$ Nantong Textile Institute, Soochow University, Nantong, People's Republic of China
}

\section{ABSTRACT}

This paper presents a study of the amidoxime/Europium (III)-chelated complex (AECC) fibers which can be used as functional fibers for technical textiles such as decorations, protections and medical textiles etc. First, the $\mathrm{EuCl}_{3}$ solution were prepared and reacted with amidoxime fibers using optimized process conditions at $50{ }^{\circ} \mathrm{C}, 5$ hours. Subsequently, the morphology, element analysis, fluorescence and mechanical properties of the AECC fibers were systematically investigated. Finally, the effects of different $\mathrm{pH}$ values and temperature on the content of Eu (III) complexes fiber were studied, respectively. The results showed that the AECC fibers possess enhanced fluorescence intensity and good mechanical properties. The results also illustrated that coordination reaction was identified between Eu (III) ions and amidoxime fiber and the electronic energy of $\mathrm{C} 1 \mathrm{~s}, \mathrm{O} 1 \mathrm{~s}, \mathrm{~N} 1 \mathrm{~s}$ and $\mathrm{Eu}$ (III) ions in amidoxime fibers has significant changes compared to original fibers. The fluorescence emission bands of the AECC fibers were observed when the excitation wavelength $\lambda$ equal to $380 \mathrm{~nm}$. In conclusion, this study provided a theoretical basis for the preparation and practical application of fluorescent fibers.

Keywords: Amidoxime/Europium (III)-chelated, complex fibers, fluorescence, mechanical properties.

\section{INTRODUCTION}

The rare earth luminous fiber has been increasingly used as functional fibers for technical textiles such as decorations, protections and medical textiles etc. The rare earth ions such as Sm (III), Eu (III), Tb (III), Dy (III) possess good fluorescence properties due to their special structural characteristics [1-4] . Chelating the amidoxime fibers with rare earth ions can produce amidoxime/Europium (III)-chelated complex (AECC) fibers which have excellent light-emitting intensity, good mechanical properties, and can free from harmful radiation $[5,6]$. They can absorb ultraviolet or visible light for ten minutes, and then can emit light continually for more than ten hours after removal of excitation resource [ $\underline{7}, \underline{8}]$. Such functional fibers can be used for technical textiles such as decorations, protections and medical textiles etc [ $\underline{5}, \underline{9}-\underline{11}]$. However, few studies have been found about using chelating reaction method to fabricate AECC fibers.

The objective of this paper is to develop amidoxime/Europium (III)-chelated complex (AECC) fibers which have enhanced fluorescence intensity and good mechanical properties. First, the $\mathrm{EuCl}_{3}$ solution were prepared and reacted with amidoxime fibers using optimized process conditions. The amidoxime fibers were made from polyacrylonitrile (PAN) polymer which can be used in many applications such as electrospinning 
fibers, carbon fibers and films due to its good anti-oxidant and chemical stability $[12,13,14]$. Subsequently, the morphology, element analysis, fluorescence and mechanical properties of the AECC fibers were systematically investigated. Finally, the effects of different $\mathrm{pH}$ values and temperature on the content of Eu (III) complexes fiber were studied, respectively.

\section{MATERIALS AND METHODS}

\subsection{Materials}

Polyacrylonitrile (PAN) fiber was purchased from Anqing Petrochemical Plant; the water used for testing is deionized water; the amidoxime fiber was prepared using the previous method [15]; $\mathrm{Eu}_{2} \mathrm{O}_{3}$, Hydroxylamine hydrochloride Sodium, Ethylenediaminetetraacetic acid disodium salt, Calcium carbonate and other reagents were of analytical grade.

\subsection{Preparation of Amidoxime/Europium (III)-chelated complexes fiber}

Adding $0.5030 \mathrm{~g}$ of $\mathrm{Eu}_{2} \mathrm{O}_{3}$ weight by analytical balance into a clean, dry small beaker, and dissolved into 15 $\mathrm{ml}$ of concentrated hydrochloric acid, then heating by water bath until the solution was transparent and $\mathrm{Eu}_{2} \mathrm{O}_{3}$ was completely dissolved. Stop heating until white crystals appear, remove the beaker and cool it at room temperature. Then dissolve the crystals in distilled water, and transfer it into $250 \mathrm{ml}$ volumetric flask to constant volume and europium chloride solution was obtained.

Weighting above $1.0 \mathrm{~g}$ amidoxime fiber into a $100 \mathrm{ml}$ of $\mathrm{EuCl}_{3}$ solution with certain concentration and adjust the $\mathrm{pH}$ value of the solution, place it into a pot with a constant temperature water bath of $50{ }^{\circ} \mathrm{C}$. After reacting about 5 hours, AECC fibers were prepared and then washed using $90 \mathrm{ml}$ of deionized water for 10 times. The lotion and the rare earth ions solution after coordination reaction were blended together and then transferred into a $1000 \mathrm{ml}$ of volumetric flask. Then determinate the concentrations of Eu (III) solution with method of complex metric titration, and calculate the content (T) of Eu (III) in AECC fibers using Equation (1). Choosing different $\mathrm{pH}$ values can obtain different AECC fibers with different colors.

$$
T=\left(C_{0} V_{0}-C_{1} V_{l}\right) \times M / m(m g / g)
$$

Where: $\mathrm{C}_{0}$ is the concentration of $\mathrm{Eu}$ (III) solution before reaction (mmol/L); $\mathrm{V}_{0}$ reprents the volume of $\mathrm{Eu}$ (III) solution before reaction, $\mathrm{V}_{0}=0.1 \mathrm{~L} ; \mathrm{C}_{1}$ is the concentration of $\mathrm{Eu}$ (III) solution after reaction $(\mathrm{mmol} / \mathrm{L}) ; \mathrm{V}_{1}$ reprents the molecular weight of Eu (III) at $151.965 \mathrm{~g} / \mathrm{mol} ; \mathrm{m}$ is PAN mass $(\mathrm{g})$.

\subsection{Characterization of fluorescence and mechanical properties}

The fluorescence spectra of AECC fibers were measured using HITACHIF-4500 fluorescence spectrometer. The tensile properties of AECC fibers were tested using LLY-068 electronic single fiber strength tester. The breaking strength and elongation at break of the fibers were repeated for 50 times by electronic single fiber strength tester and all data sets were expressed in terms of their mean and standard deviation.

\subsection{X-ray photoelectron spectroscope}

The samples were characterized with an X-ray photoelectron spectroscope (XPS) (Escalab MKII, British,Vaccum Generator Co., Ltd.). Analyses were performed on a surface analysis system with a MgK X-ray source, vacuum rate $1.33 \times 10-7 \mathrm{~Pa}, 10$ scans. Samples were attached to the aluminum sample platform with double-sided tapes. Quantitative analyses were performed using peak areas and elemental sensitivity factors. Mean values were gotten from five different areas on one sample.

\section{RESULTS AND DISCUSSION}

\subsection{Effects of different pH on Eu (III) content in AECC fibers}

The original color of amidoxime fibers is white. When the value of $\mathrm{pH}$ was 1.0 , the color of the fibers did not change significantly. As the $\mathrm{pH}$ value increased, the color of fiber gradually deepened, while the value of $\mathrm{pH}$ was above 5.0, the fiber color faded.

As shown in Table 1, when $\mathrm{pH}$ was equal to 1.0, the fiber was white. Because of the high acidity, the 
groups of amidoxime fiber $-\mathrm{NH} 2$ and $-\mathrm{OH}$ changed to be $-\mathrm{NH} 3$ and $-\mathrm{OH} 2$ respectively, and lost the ability to be coordinated reaction with $\mathrm{Eu}$ (III). The color of fiber deepened as the acidity decreased at $\mathrm{pH}<3$, and it showed that the content of Eu (III) in the fiber increased. The color of fiber faded as the acidity decreased and the content of $\mathrm{Eu}$ (III) in the fiber significantly reduced while $\mathrm{pH}>4$. The color of fiber was the darkest when $\mathrm{pH}=3.0$. Meanwhile, the content of Eu (III) in the fiber also reached the highest, and the amidoxime groups and $\mathrm{Eu}$ (III) were matched into stable mating structure.

Table 1: Effect of $\mathrm{pH}$ value of reaction system on the color of fiber and Eu (III) content

\begin{tabular}{l|l|l|l}
\hline PH VALUE & COLOR OF FIBER & EU (III) CONTENT T & UNIT \\
\hline 1.0 & White & 0 & $(\mathrm{mg} / \mathrm{g})$ \\
\hline 2.0 & yellow-green (Deep) & $94.90 \pm 0.05$ & $(\mathrm{mg} / \mathrm{g})$ \\
\hline 3.0 & yellow-green (Light) & $40.56 \pm 0.03$ & $(\mathrm{mg} / \mathrm{g})$ \\
\hline 4.0 & yellow-green (Deep) & $72.57 \pm 0.14$ & $(\mathrm{mg} / \mathrm{g})$ \\
\hline 5.0 & yellow-green (Light) & $50.59 \pm 0.03$ & $(\mathrm{mg} / \mathrm{g})$ \\
\hline
\end{tabular}

\subsection{Effects of different temperature on Eu (III) content in AECC fibers}

After blending $0.2 \mathrm{~g}$ amidoxime fibers with $25 \mathrm{ml} \mathrm{Eu}$ (III) solution in five erlenmeyer flasks, the $\mathrm{pH}$ of the solution was adjusted to 3.0 , and then were placed at $20{ }^{\circ} \mathrm{C}, 25{ }^{\circ} \mathrm{C}, 35{ }^{\circ} \mathrm{C}, 45^{\circ} \mathrm{C}$ and $55^{\circ} \mathrm{C}$ to reaction for 5 hours, respectively. Finally, the fibers were removed and washed using deionized water. So different content AECC fibers were obtained. The Eu (III) content in AECC fibers under different reaction temperatures was calculated using Equation (1). The calculated results are given in Table 2.

Table 2: Effect of the reaction temperature on the color of fiber and the content of Eu (III) fiber

\begin{tabular}{l|l|l|l}
\hline REACTION TEMPERATURE & COLOR OF FIBER & EU (III) CONTENT & UNIT \\
\hline $20^{\circ} \mathrm{C}$ & Light yellow & $5.65 \pm 0.02$ & $(\mathrm{mg} / \mathrm{g})$ \\
\hline $25^{\circ} \mathrm{C}$ & Light yellow & $6.67 \pm 0.03$ & $(\mathrm{mg} / \mathrm{g})$ \\
\hline $35^{\circ} \mathrm{C}$ & yellow-green (Light) & $23.55 \pm 0.05$ & $(\mathrm{mg} / \mathrm{g})$ \\
\hline $45^{\circ} \mathrm{C}$ & yellow-green (Deep) & $90.69 \pm 0.05$ & $(\mathrm{mg} / \mathrm{g})$ \\
\hline $55^{\circ} \mathrm{C}$ & yellow-green (Light) & $60.95 \pm 0.04$ & $(\mathrm{mg} / \mathrm{g})$ \\
\hline
\end{tabular}

Temperature was an important factor affecting the reaction rate of PAN fibers and Eu (III) ion. Generally, the reaction rate increased as the increasing of temperature. As the data given in Table 1, the content of Eu (III) in AECC fibers increased significantly while the temperature changed to $35^{\circ} \mathrm{C}$, and the color deepened obviously. Little change of the content was observed at the lower temperatures. When the measured temperature was reached at $45{ }^{\circ} \mathrm{C}$, the Eu (III) content in AECC fibers reached at maximum. While the measured temperature was increased to $55{ }^{\circ} \mathrm{C}$, the $\mathrm{Eu}$ (III) content in the fibers decreased rapidly. Meanwhile, the color of fiber became lighter. Thus, the high temperature could destroy the structure of the fiber and decrease the mechanical properties.

\subsection{XPS analysis of AECC fibers}

As the data given in Table 3, a C1s binding energy had significantly changed in AECC fiber. The binding energy of $\mathrm{C} 1 \mathrm{~s}$ in amidoxime fibers was $286.14 \mathrm{eV}$, the binding energy of C1s in AECC was $286.09 \mathrm{eV}$. It demonstrated that the binding energy of carbon in $-\mathrm{C}=\mathrm{N}$ - was changed. The binding energy of $\mathrm{O} 1 \mathrm{~s}$ in amidoxime fibers was $532.37 \mathrm{eV}$, the binding energy of O1s in AECC fibers was $532.00 \mathrm{eV}$. The reason is that a coordination bond was formed between the $-\mathrm{OH}$ groups in amidoxime fibers and the rare earth ions Eu (III), and the oxygen during new chemical environment was produced. Meanwhile, the bonded hydrogen state became the coordination state with the rare earth ion Eu (III), hence the bonds were strengthened. The combined IR analysis and significant changes of the fibers color before and after the reaction mutually demonstrated the coordination of $-\mathrm{OH}$ group and the rare earth ions Eu (III). The binding energy of N1s in AECC fibers increased slightly to $399.33 \mathrm{eV}$ compared to the binding energy of N1s in amidoxime fibers (399.26 
$\mathrm{eV}$ ). It demonstrated that the coordination had occurred between $\mathrm{N}$ in $-\mathrm{N}_{2} \mathrm{H}$ - and rare earth ions. Meanwhile, the electron energy of rare earth ions Eu (III) were all changed, which proved that the rare earth ions involved in the coordination reaction.
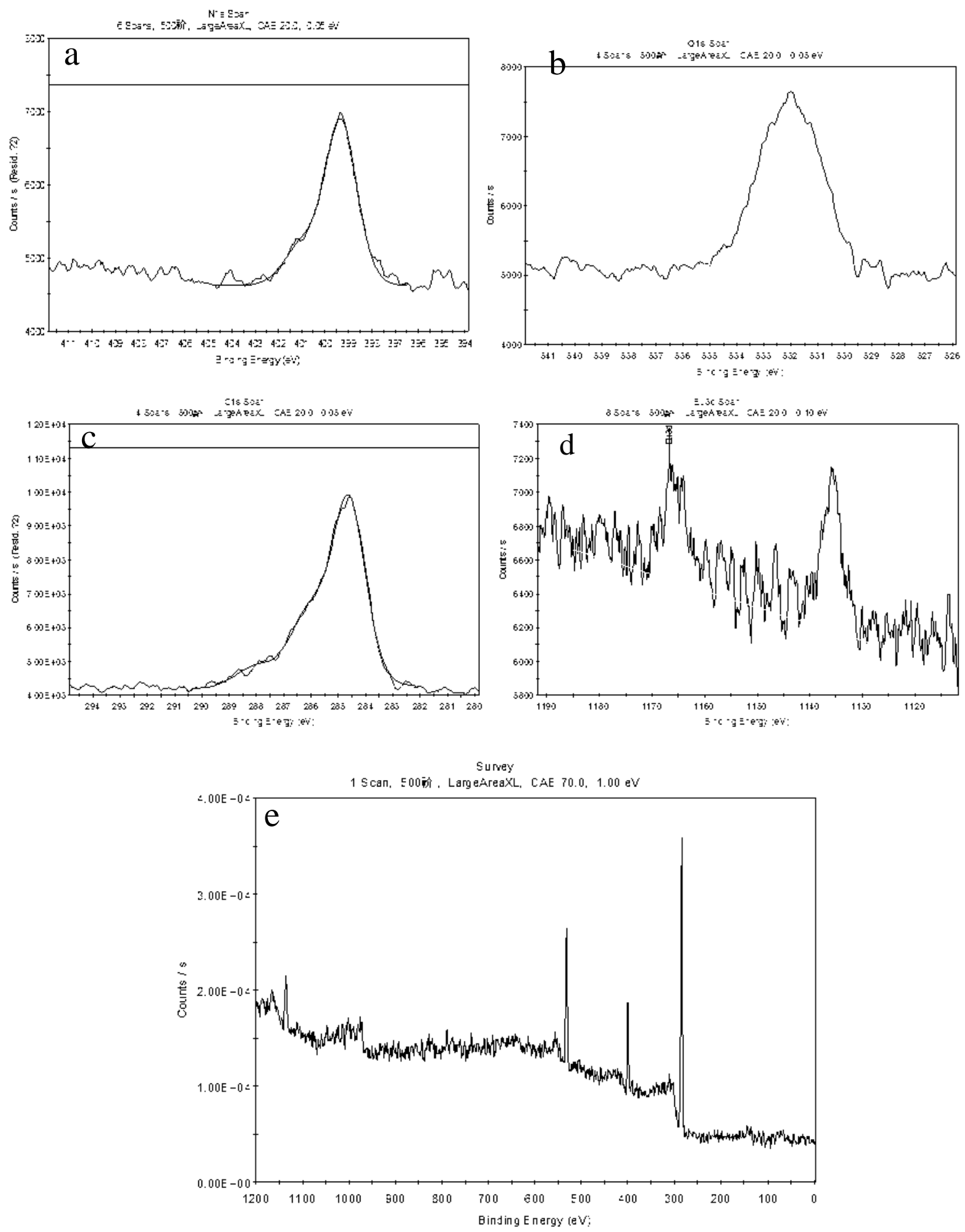

Figure 1: XPS spectras of different elements and AECC fibers: a - e are the bind ing energies of C1s, O1s, N1s, Eu (III) elements and AECC fibers 
Table 3: The binding energy of different elements and AECC fibers

\begin{tabular}{l|l|l}
\hline RARE EARTH IONS & AMIDOXIME FIBERS & AECC FIBERS \\
\hline $\mathrm{C} 1 \mathrm{~s}$ & 284.14 & 284.09 \\
\hline $\mathrm{O} 1 \mathrm{~s}$ & 532.37 & 532.00 \\
\hline $\mathrm{N} 1 \mathrm{~s}$ & 399.26 & 399.33 \\
\hline $\mathrm{Eu}(\mathrm{III})$ & -- & 1135.7 \\
\hline
\end{tabular}

\subsection{Morphology of AECC fibers}

In Figure 2, the scanning electron microscope (SEM) photograph of the PAN fiber surface is uniform and smooth and has no obvious defects. While the surface of amidoxime fiber after reaction with hydroxylamine solution maintained a relatively smooth apparent morphology, some uniform rough dents were observed, which showed the apparent of PAN fiber changed after amidoxime. Hence, the emergences of a number of defects were induced on the surface of the fibers. AECC fibers preduced by the reaction of Europium (III) solution had rougher surface and more significant jagged dents, which showed that amidoxime fibers and rare earth solution had a full reaction, and the surface of the fibers was coordinated a large amount of rare earth ions. And they were uniformly distributed onto the surface of the fibers; meanwhile, it also showed the mechanical properties of fiber were affected, so it's breaking strength and elongation at break decreased.

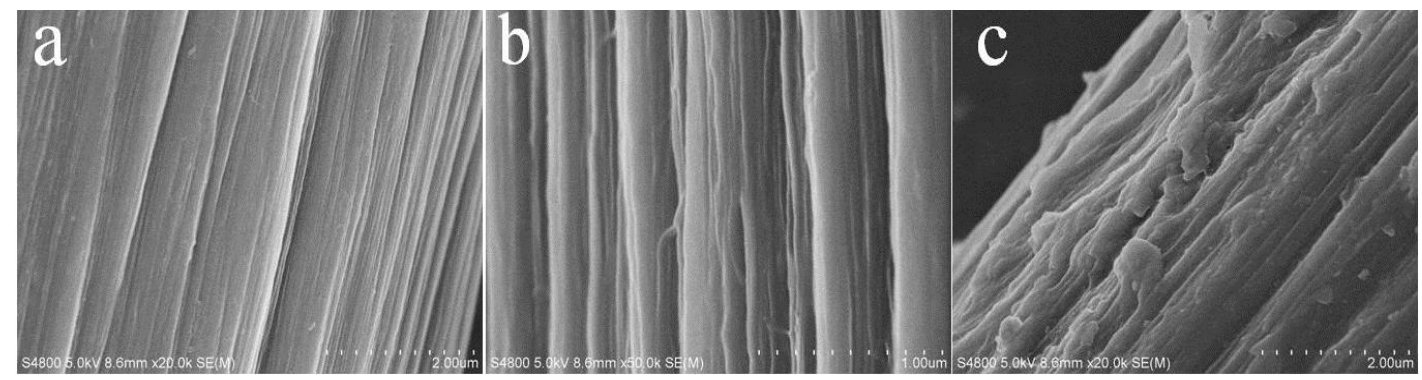

Figure 2: SEM images of PAN fibers (a), amidoxime fibers (b) and AECC fibers (c).

\subsection{Fluorescence spectra of AECC fibers}

The electronic arrangement formula of $\mathrm{Eu}$ (III) is $4 \mathrm{f}^{7} 6 \mathrm{~s}^{2}$, the $\mathrm{f}$ shell electron of its secondary outer layer electron is unfilled, and it belongs to be paramagnetic. In addition, the excited state of the ion ( $\mathrm{m} *$ ) energy layer (i.e. the $\mathrm{f} *$ energy layer which is transferred from $\mathrm{f}$ energy layer) located below the lowest excited state energy layer of the ligand, and there are no multi energy layers between $\mathrm{m} *$ and $\mathrm{s}_{0}$ energy layers. Thus, the ions can emit linear characteristic luminescence. The spectrum was determined under the conditions of $10 \mathrm{~nm}$ incident and emission slit at room temperature. The characteristic peaks of AECC fibers appeared at the excitation wavelength of $380 \mathrm{~nm}$, and AECC fibers appeared two bands, either of which the $469 \mathrm{~nm}$ luminescence bands might be ligand luminescence emission and it was luminescence emission band of Eu (III) at the $616 \mathrm{~nm}$. 


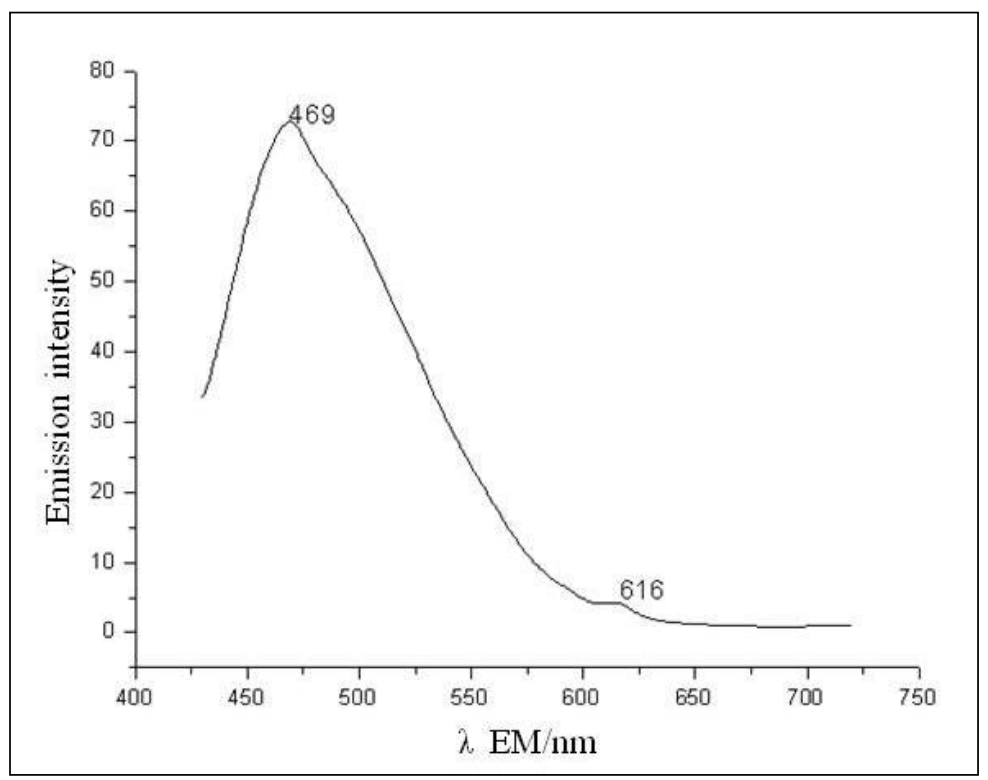

Figure 3: fluorescence spectra of AOCF-Eu (III) complexes fiber

\subsection{Mechanical properties of AECC fibers}

The breaking strength and elongation at break of fiber samples were measured for 50 times using electronic single fiber strength tester (LLY-068). The breaking strength and elongation at break of PAN fibers were $0.71 \mathrm{cN}$ and $18.29 \%$, their sample variances were 0.0017 and 2.47 , respectively. The breaking strength and the elongation at break of amidoxime fibers were $0.68 \mathrm{cN}$ and $19.86 \%$, their sample variances were 0.0026 and 3.31, respectively. The breaking strength of amidoxime fibers was lower than that of the acrylic fiber (PAN). The reason is that after amidoxime reaction of the $-\mathrm{CN}$ in PAN, the polymer chain on the appearance of fiber became uneven and part of the polymer chain fractured, so the surface tension of the fiber declined while elongation at break increased. The fibers became thinner, the elastic of fibers became better, and the sample variance of test values before and after the reaction became larger, data became scattered. It illustrated that the unstable properties of the fibers maybe induced by the damage of fiber during the process of reaction.

The relationships among different Eu (III) content and breaking strength and elongation at break of fiber were derived (Table 4). As can be seen from Table 4, the breaking strength and elongation at break of fiber decreased with the increase of content of $\mathrm{Eu}$ (III). There are two reasons. First, when amidoxime groups coordinated with $\mathrm{Eu}$ (III), a body structure can be formed because one Eu (III) ion can coordinate with two amidoxime groups on different strand of fiber, so the elastic of the fibers decreased. Second, soaking solution with different $\mathrm{pH}$ and temperature can cause the swelling and corrosion role on the fiber, and some physical adsorption also destroyed the fiber polymer structure, hence the elasticity of original fibers was decreased. Compared to the PAN fibers, the breaking strength and elongation of AECC fibers were all decreased with the increase of the content of Eu (III) ion. But, the produced AECC fibers possess a good ability for yarn spinning.

Table 4: The relations among different content of Eu (III) and tensile property of AECC fibers

\begin{tabular}{c|c|c}
\hline CONTENT OF EU (III) /(MG. G-1) & BREAKING STRENGTH /CN & ELONGATION AT BREAK /\% \\
\hline 5.65 & 0.695 & 18.30 \\
\hline 6.67 & 0.612 & 17.93 \\
\hline 23.55 & 0.603 & 17.56 \\
\hline 60.95 & 0.584 & 16.83 \\
\hline 90.69 & 0.522 & 16.27 \\
\hline
\end{tabular}

Note: $\mathrm{A}$ is Eu (III) (mg) / amidoxime fiber (g) 


\section{CONCLUSIONS}

In this work, the AECC fibers were successfully prepared by chelating reaction using amidoxime fibers (made from PAN fibers) and Europium oxide. The highest Eu (III) content can be harvest when the optimum reaction condition was set at $\mathrm{pH}=3.0$ and temperature $=45^{\circ} \mathrm{C}$, time $=5$ hours. Meanwhile, the fluorescence of the AECC fibers was the highest among other groups according to the results of a series of measurements. The emission intensity of AECC fibers increased as the increasing content of Eu (III). When the amidoxime group fully reacted, the AECC fibers would occurred "concentration quenching" phenomenon with the increasing content of $\mathrm{Eu}$ (III). The mechanical properties of AECC fibers declined as the increasing content of $\mathrm{Eu}$ (III). In conclusion, this study provided a theoretical basis for the preparation and practical application of fluorescent fibers.

\section{ACKNOWLEDGEMENT}

The work is supported by Priority Academic Program Development of Jiangsu Higher Education Institutions (PAPD) , National Natural Science Foundation of China under grant Nos. 20771002 \& 11372205 and Project for Six Kinds of Top Talents in Jiangsu Province under grant No. ZBZZ-035, Science \& Technology Pillar Program of Jiangsu Province under grant No. BE2013072

\section{BIBLIOGRAPHY}

[1] ZHANG, J., GE, M. Q., "A study of an anti-counterfeiting fiber with spectral fingerprint characteristics", Journal of the Textile Institute, v.102, pp.767-773, 2011.

[2] SUJATHA, N., MURUKESHAN, V., ONG, L., et al., "An all fiber optic system modeling for the gastrointestinal endoscopy: design concepts and fluorescent analysis", Optics communications, v. 219, pp. 71-79, 2003.

[3] CHEN, M., QU, H., ZHU, J., et al. "Magnetic electrospun fluorescent polyvinylpyrrolidone nanocomposite fibers", Polymer, v. 53, pp. 4501-4511, 2012.

[4] SANTOS, M. dos, FREIRE, C., "Study of the performance of films containing rare earth salts as corrosion inhibitors of zinc-iron alloy electroplated steel", Matéria (Rio de Janeiro), v. 17, pp. 1144-1157, 2012.

[5] YAN, Y., GE, M.Q., LI, Y., et al., "Morphology and spectral characteristics of a luminous fiber containing a rare earth strontium aluminate", Textile Research Journal, v. 82, pp. 1819-1826, 2012.

[6] ZHANG, J., GE, M. Q., "Effects of transparent inorganic pigment on spectral properties of spectrumfingerprint anti-counterfeiting fiber containing rare earths", Journal of Rare Earths, v. 30, pp. 952-957, 2012.

[7] ZHU, Y., GE, M., CHEN, Z., "Preparation and properties of rare earth luminous fiber containing red organic fluorescent pigment", Journal of Rare Earths, v. 31, pp. 1043-1048, 2013.

[8] GUO, X., GE, M., ZHAO, J., "Photochromic properties of rare-earth strontium aluminate luminescent fiber", Fibers and Polymers, v. 12, pp. 875-879, 2011.

[9] GONCALVES, H.M., DUARTE, A. J., DAVIS, F., et al., "Layer-by-layer immobilization of carbon dots fluorescent nanomaterials on single optical fiber". Analytica chimica acta, v. 735, pp. 90-95, 2012.

[10] HUSSAIN, M., SHAMEY, R., HINKS, D., et al., "Synthesis of novel stilbene-alkoxysilane fluorescent brighteners, and their performance on cotton fiber as fluorescent brightening and ultraviolet absorbing agents", Dyes and Pigments. v. 92, pp. 1231-1240, 2012.

[11] MOTHUDI, B., NTWAEABORWA, O., KUMAR, A., et al., "Phosphorescent and thermoluminescent properties of $\mathrm{SrAl} 2 \mathrm{O}$ 4: Eu 2+, Dy 3+ phosphors prepared by solid state reaction method" Physica B: Condensed Matter. v. 407, pp.1679-1682, 2012.

[12] WAN, Y. Q., HE, J. H., WU, Y., YU, J. Y., "Vibrorheological effect on electrospun polyacrylonitrile (PAN) nanofibers", Materials Letters, v. 60, pp. 3296-3300, 2006.

[13] KONG, H.Y., He, J.H., et al., Polymer liquid membrane for nanofiber fabrication, Thermal Science, v. 17, n. 5, pp. 1479-1482, 2013

[14] WANG, P., HE, J.H. Electrospun polyvinyl alcohol-milk nanofibers, Thermal Science, v. 17, n. 5, pp. $1515-1516,2013$ 
HU, C. G.; WANG, S.L.; WU, Z.Z.; ZAO, Z.; LI, G. revista Matéria, v. 19, n. 4, pp. 350-357, 2014.

[15] TAO, T. X., WU, Z. Z., ZHAO, Z. Q., "Preparation of chelating fibers - modification of polyacrylonitrile fiber", Synthetic Fiber in China, v. 4, pp. 32-33, 2001. 